\title{
Tranexamic Acid Reduces Postoperative Blood Loss in Distal Femoral Osteotomy
}

\author{
Michael E. Steinhaus, MD ${ }^{1} \quad$ Joshua Buksbaum, BS ${ }^{1}$ Avraham Eisenman, BA ${ }^{1}$ Monal Kohli, MS ${ }^{1}$ \\ Austin T. Fragomen, $\mathrm{MD}^{1} \quad$ S. Robert Rozbruch, $\mathrm{MD}^{1}$ \\ ${ }^{1}$ Limb Lengthening and Complex Reconstruction Service, \\ Address for correspondence Michael E. Steinhaus, MD, Limb \\ Department of Orthopaedic Surgery, Hospital for Special Surgery, \\ New York, New York \\ Lengthening and Complex Reconstruction Service, Department of \\ Orthopaedic Surgery, Hospital for Special Surgery, 535 East 70th \\ Street, New York, NY 10021 (e-mail: steinhausm@hss.edu).
}

J Knee Surg 2020;33:440-444.

\begin{abstract}
Keywords

- postoperative blood loss

- tranexamic acid

- distal femoral osteotomy

- deformity
\end{abstract}

Blood loss remains a significant source of morbidity and mortality in orthopaedic surgery, with transfusions associated with an increased risk of infection, length of stay, delayed rehabilitation, and significantly increased hospitalization costs. The purpose of this study was to assess whether the use of tranexamic acid (TXA) is effective in reducing postoperative blood loss in patients undergoing distal femoral osteotomy (DFO). A retrospective review was performed of all patients undergoing DFO by a single surgeon from 2010 to 2017, with a change in protocol occurring in 2014, after which all patients received TXA. Patients in the TXA group $(n=24)$ received 1-g TXA immediately prior to incision followed by a second dose of 1-g TXA 4 hours after the administration of the first dose. Patients in the control group $(n=28)$ did not receive TXA. Drainage was recorded through a subfascial drain that remained for 24 hours postoperatively. Postoperative hemoglobin, hematocrit, and transfusions, as well as demographic factors, including age, gender, body mass index (BMI), medical comorbidities, and ASA (American Society of Anesthesiologists) class, were recorded. Multivariate regression analysis adjusting for potential confounding variables was performed. With the exception of gender, the two groups did not differ significantly in baseline characteristics, including age, BMI, and ASA class. There was a significant difference in postoperative blood loss, with those receiving TXA having a mean drain output of 184.2 versus $242.1 \mathrm{~mL}$ for the control group ( $p=0.02$ ), which persisted after regression analysis $(p<0.005)$. Blood loss differed between patients who received one $(250 \mathrm{~mL})$ dose and those who received two $(162.2 \mathrm{~mL})$ doses of TXA, although this difference was insignificant $(p=0.489)$. There were no differences in postoperative hemoglobin and hematocrit levels. One patient (control group) required blood transfusion postoperatively. There were no complications related to TXA. In conclusion, TXA results in less postoperative blood loss in DFO, with the most pronounced effect in those who receive two doses. Future research should involve a larger, prospective study to assess for differences in postoperative hemoglobin/hematocrit levels and transfusion rates. received

September 15, 2018

accepted after revision

December 27, 2018

published online

February 12, 2019
Copyright (c) 2020 by Thieme Medical

Publishers, Inc., 333 Seventh Avenue, New York, NY 10001, USA. Tel: +1(212) 760-0888
DOI https://doi.org/ 10.1055/s-0039-1678540. ISSN 1538-8506. 
Blood loss remains a significant source of morbidity and mortality in orthopaedic surgery, with transfusions associated with an increased risk of bacterial infection, length of stay, delayed rehabilitation, and significantly increased hospitalization costs. ${ }^{1-9}$ In the trauma setting, blood transfusions are also associated with decreased survival and increased risk of mortality $>90$ days after hip fracture surgery. ${ }^{10}$ In the past, blood transfusions have been quite common postoperatively, with transfusions required in 11 to $67 \%$ of arthroplasties, ${ }^{11,12} 2$ to $36 \%$ of spine surgery, ${ }^{4,13,14} 20$ to $60 \%$ of hip fractures, and $100 \%$ of patients requiring intramedullary nailing for two or more long bone fractures. ${ }^{15-17}$ Transfusion requirement in limb realignment at the distal femur is not well documented in the literature, although one study noted a transfusion rate of $31 \%$ associated with distal femur fractures in the elderly. ${ }^{18}$

While many strategies have been trialed to reduce blood loss in orthopaedic surgery, tranexamic acid (TXA) has emerged as an inexpensive and effective way to prevent transfusions in a variety of settings. Surgical bleeding is thought to be enhanced by the activation of local fibrinolysis factors, particularly in the setting of a pneumatic tourniquet. ${ }^{19}$ TXA, a synthetic lysine analogue, reversibly binds plasminogen, preventing its conversion to plasmin and thus the proteolytic action of degrading fibrin. ${ }^{20}$ The benefit of TXA has been acknowledged across multiple surgical disciplines and is used in cardiac surgery, gynecological surgery, and neurosurgery, among others. In orthopaedics, TXA has been most tested in arthroplasty, with numerous studies demonstrating its benefit in total knee arthroplasty (TKA) $)^{21-26}$ and total hip arthroplasty (THA). ${ }^{27-30}$ In the trauma setting, early studies suggest that TXA is safe and effective. In the CRASH-2 (Clinical Randomization of an Antifibrinolytic in Significant Hemorrhage 2) study, more than 20,000 patients were randomized to TXA or placebo, with patients receiving TXA having reduced all-cause mortality and risk of death due to bleeding. ${ }^{31,32} \mathrm{~A}$ recent systematic review reported on TXA use in hip fracture surgery, reporting that intravenous (IV) TXA significantly reduced the blood transfusion requirement without increased risk of thromboembolic (TE) events. ${ }^{33}$

While TXA has demonstrated efficacy in these contexts, we know of no prior study assessing its utility in limb deformity. The purpose of this study is to evaluate the benefit of TXA in distal femoral osteotomy (DFO). We hypothesize that patients receiving TXA will have lower postoperative blood loss compared with controls. Establishing the efficacy of TXA in DFO for limb realignment will benefit patients, surgeons, hospitals, and payers alike.

\section{Methods}

Institutional Review Board approval was obtained prior to initiation of the study. A retrospective review was performed on all patients undergoing DFO by a single author (S.R.R.) from 2010 to 2017. For each case, the same protocol was followed. A tourniquet was inflated prior to incision and deflated after skin closure. A deep, subfascial drain was left in place and removed 24 hours postoperatively, and postoperative drainage was recorded. For the senior author, a change in protocol occurred in April 2014, when patients began to receive one dose of 1-g TXA immediately prior to incision ( \pm a second dose of 1-g TXA 4 hours following administration of the first dose).

A total of 28 patients underwent DFO without TXA between 2010 and 2014 and were included in the control group. A total of 24 patients underwent DFO with TXA between 2014 and 2017 and were included in the TXA group (6 patients received one dose, 18 patients received two doses). Demographic factors including age, gender, body mass index (BMI), medical comorbidities, and ASA (American Society of Anesthesiologists) class were recorded. The primary outcome measure included total postoperative blood loss as indicated by drain output. Secondary outcomes included postoperative hemoglobin and hematocrit values and postoperative transfusion rate.

\section{Statistical Analysis}

Demographic variables were compared using Student's $t$-test and chi-square test. A generalized linear regression model was used to compare the blood loss between the two groups, controlling for potential confounding factors including age, BMI, gender, and ASA class. All analyses were performed using SAS statistical software (SAS Institute Inc., Cary, NC).

\section{Results}

A total of 52 patients met inclusion criteria and were included in the study. Demographic details of the included patients are shown in - Table 1. Mean age for the control and TXA groups were 40.4 and 41.7 years, respectively. The two groups did not differ significantly in baseline characteristics including age, BMI, and ASA class. The gender distribution did differ significantly, with 5 males and 23 females in the control group compared with 12 males and 12 females in the TXA group $(p=0.0138)$.

Postoperative blood loss, and pre- and postoperative hemoglobin and hematocrit levels were assessed and are shown in - Fig. 1A-C. There was a significant difference in postoperative blood loss, with those receiving TXA (one dose or two doses) having a mean drain output of $184.2 \mathrm{~mL}$ (range: $0-500 \mathrm{~mL}$ ) compared with $242.1 \mathrm{~mL}$ (range: $50-530 \mathrm{~mL}$ ) for the control group $(p=0.02)$. This significant difference persisted after regression analysis $(p<0.005)$. There were no significant differences in postoperative hemoglobin and hematocrit levels between patients receiving TXA and the controls.

Table 1 Patient demographics

\begin{tabular}{|l|l|l|l|}
\hline & $\begin{array}{l}\text { Control } \\
(\boldsymbol{n}=\mathbf{2 8})\end{array}$ & TXA $(\boldsymbol{n}=\mathbf{2 4})$ & $p$-Value \\
\hline Age (years) & $40.36 \pm 16.35$ & $41.71 \pm 17.18$ & 0.7729 \\
\hline BMI $\left(\mathrm{kg} / \mathrm{m}^{2}\right)$ & $29.12 \pm 5.96$ & $26.76 \pm 4.97$ & 0.1318 \\
\hline $\begin{array}{l}\text { Sex } \\
\text { (male:female) }\end{array}$ & $5: 23$ & $12: 12$ & 0.0138 \\
\hline $\begin{array}{l}\text { ASA class } \\
(\mathrm{I} / \mathrm{II} / \mathrm{III})\end{array}$ & $7 / 18 / 3$ & $4 / 19 / 1$ & 0.5295 \\
\hline
\end{tabular}

Abbreviations: ASA, American Society of Anesthesiologists; BMI, body mass index; TXA, tranexamic acid. 


\section{Mean Postoperative Blood Loss}
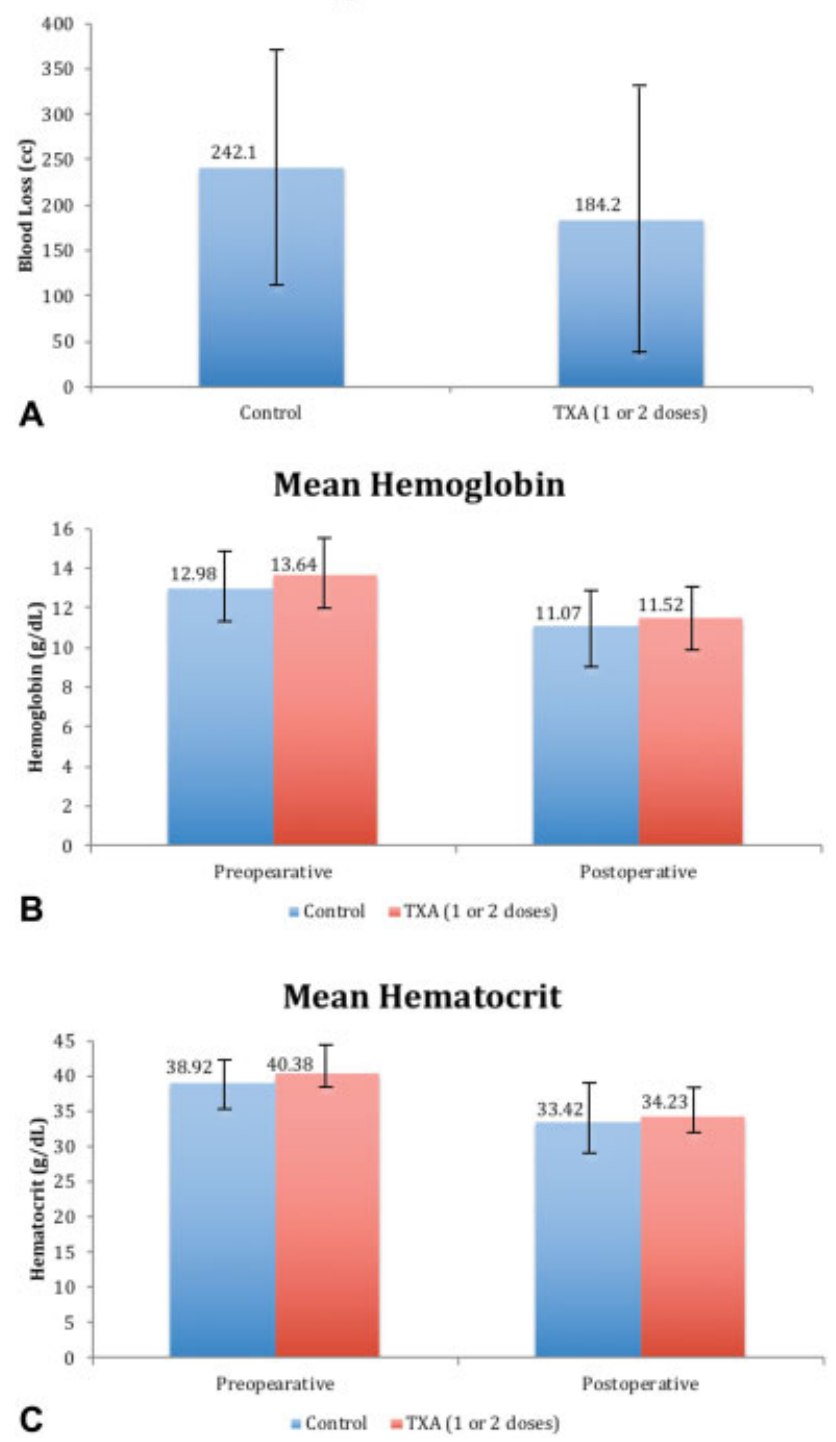

Fig. 1 (A) Mean postoperative blood loss: there was a significant difference in postoperative blood loss, with those receiving tranexamic acid (TXA; one dose or two doses) having a mean drain output of $184.2 \mathrm{~mL}$ compared with $242.1 \mathrm{~mL}$ for those in the control group $(p=0.02)$. (B) Mean preoperative and postoperative hemoglobin: no significant difference was found in preoperative or postoperative hemoglobin values between controls and those who received TXA (one dose or two doses). (C) Mean preoperative and postoperative hematocrit: no significant difference was found in preoperative or postoperative hematocrit values between controls and those who received TXA (one dose or two doses).

When stratified by dosing regimen, those receiving two doses of TXA had a significant decrease in postoperative blood loss (162.2 mL) compared with the controls $(242.1 \mathrm{~mL}$; $p=0.014)$, although this difference was not present for patients who received only one dose of TXA $(250 \mathrm{~mL})$. No differences were found in hemoglobin or hematocrit levels when stratified by dosing regimen. One patient in the control group required blood transfusion postoperatively, whereas no patients in the TXA group required transfusion. There were no complications, including deep venous thrombosis, related to the use of TXA.

\section{Discussion}

The results of our study demonstrate that the administration of TXA decreases postoperative blood loss in the setting of DFO. Specifically, patients receiving TXA had a mean postoperative blood loss approximately $25 \%$ less than patients who did not receive TXA. When stratified by dosing, we found that patients who received two doses of TXA had significantly less blood loss than those in the control group, whereas patients who received only one dose of TXA intraoperatively showed no such difference.

TXA has been studied extensively, consistently demonstrating efficacy in the hip and knee arthroplasty literature. In a meta-analysis of randomized controlled trials (RCTs) in primary TKA, Yang et al reported a decrease in blood loss of $504 \mathrm{~mL}$ and reduction in transfusion by 1.43 units per patient associated with the use of IV TXA. ${ }^{26}$ Similarly, in their metaanalysis of RCTs studying TXA use in THA, Sukeik et al noted a reduction in total blood loss of $289 \mathrm{~mL}$ and a significant reduction in transfusion rate for patients receiving TXA. ${ }^{34}$ Reducing the transfusion rate has had a transformational effect in arthroplasty. Prior to the popularization of TXA in arthroplasty, allogeneic transfusions were quite common, with transfusion rates of 11 to $67 \%,{ }^{11,12}$ whereas a recent study on IV and topical TXA in TKA reported transfusion rates of 0.6 and $1.6 \%$, respectively. ${ }^{35}$

The appropriate dose of TXA in DFO remains to be seen. According to the results of our study, patients who received two doses of TXA had significantly lower blood loss than those in the control group, whereas those who received only one dose did not. Several others have studied TXA dosing, generally reporting increased effect with higher doses of TXA. Alshryda et a ${ }^{36}$ performed a meta-analysis of RCTs in primary TKA, with subgroup analysis demonstrating that high-dose ( $>4 \mathrm{~g}$ ) TXA may be significantly associated with reduction in blood transfusion (risk ratio: 5.33). Similarly, Tanaka et al found that TXA had the greatest effect in reducing blood loss and maintaining greater postoperative hemoglobin in TKA when it was given preoperatively and again on deflation of the tourniquet compared with a single dose of TXA, ${ }^{25}$ and Maniar et al found that adjuvant doses (either preoperatively, or preoperatively and postoperatively) resulted in significantly reduced drain loss compared with controls, whereas one dose $(10 \mathrm{mg} / \mathrm{kg})$ given intraoperatively was ineffective. ${ }^{37}$ Interestingly, when analyzing total blood loss, these authors found that patients who had an additional dose of TXA preoperatively had a reduction in total blood loss compared with controls, whereas those with an additional dose postoperatively did not, suggesting that preoperative TXA administration has advantages over postoperative administration. Others, however, have found no benefit to additional TXA doses. Lin et al, for example, found no difference in total blood loss or transfusion rate between two doses (preoperative and intraoperative) compared with a single intraoperative dose in minimally invasive TKA. ${ }^{38} \mathrm{Ker}$ et al performed a meta-analysis of TXA use across multiple disciplines and found a poor dose-response relationship between TXA dose and blood loss, noting that a total dose of $1 \mathrm{~g}$ was sufficient for most adults. ${ }^{39}$ While we did detect a 
significant decrease in blood loss for the patients who received only one dose, this group was very small, comprising only six patients. Therefore, we feel that we were unable to draw conclusions about the effect of one dose of TXA based on these results and that this should be a topic of further research.

Not only has TXA shown efficacy broadly, but experience to date has also demonstrated its favorable safety profile. The risk of TE disease is the primary concern for patients who receive TXA, which was assessed in a recent study. ${ }^{40}$ Madsen et al performed a retrospective review of more 3,000 patients undergoing THA or TKA, of which the majority (87.6\%) received TXA preoperatively, and found a $1 \%$ risk of TE event, with no difference between patients who received TXA and those who did not. Within the TXA group, the authors found that higher age (odds ratio [OR]: 1.06) and particularly cardiovascular disease (OR: 4.78) were significantly correlated with the likelihood of having a TE event. Yang et al reported similar findings in a meta-analysis of RCTs in primary TKA, noting no difference in prothrombin time, activated partial thromboplastin time, deep vein thrombosis, or pulmonary embolus between TXA and placebo. ${ }^{26}$ Similarly, another group performed a meta-analysis on RCTs of TXA use in TKA and THA, reporting no increased risk for VTE associated with TXA. ${ }^{41}$ Finally, in their large retrospective cohort study of more than 800,000 hip and knee arthroplasty patients, TXA was associated with no increased risk of VTE. ${ }^{42}$ We had no such complications in our cohort, which is consistent with prior literature demonstrating the favorable safety profile of TXA.

Our study has several limitations. First, this was not a prospective study but a retrospective review and therefore is limited by potential bias conferred by this type of study. There was a difference in the baseline gender composition between the two groups that we do not expect would bias the results in either direction; otherwise, no significant baseline differences were identified. Furthermore, while this was a retrospective study, the difference in treatment represented a protocol shift in the management of these patients, where all patients after a certain date received TXA, perhaps limiting the bias of the study's retrospective nature. Second, there are limitations associated with the use of postoperative drain output as a proxy for postoperative blood loss, including small variations in how much blood collects in the drains or precise times the drains were removed, as well as the potential for the drains to clot off. Finally, we are limited by the small sample size of our cohort, specifically regarding drawing conclusions about differences in TXA dosing. A larger, prospective, randomized study would help to elucidate these differences and limit the drawbacks of our study design.

\section{Conclusions}

While TXA has been studied extensively in the arthroplasty literature, to our knowledge, this is the first study to report on the efficacy of TXA in the setting of distal femur osteotomy for limb realignment. As hypothesized, we found that TXA use is effective in reducing postoperative blood loss without associated complications. Given the small nature of our study, conclusions cannot be drawn regarding the optimal dosing in this setting, which should be studied in a larger, randomized, prospective study in the setting of DFO.

Note

Investigation was performed at the Department of Orthopaedic Surgery, Hospital for Special Surgery, New York, NY.

Conflict of Interest

None declared.

\section{References}

1 Hill GE, Frawley WH, Griffith KE, Forestner JE, Minei JP. Allogeneic blood transfusion increases the risk of postoperative bacterial infection: a meta-analysis. J Trauma 2003;54(05):908-914

2 Saleh A, Small T, Chandran Pillai AL, Schiltz NK, Klika AK, Barsoum WK. Allogenic blood transfusion following total hip arthroplasty: results from the nationwide inpatient sample, 2000 to 2009. J Bone Joint Surg Am 2014;96(18):e155

3 Shokoohi A, Stanworth S, Mistry D, Lamb S, Staves J, Murphy MF. The risks of red cell transfusion for hip fracture surgery in the elderly. Vox Sang 2012;103(03):223-230

4 Basques BA, Anandasivam NS, Webb ML, et al. Risk factors for blood transfusion with primary posterior lumbar fusion. Spine 2015;40(22):1792-1797

5 Kato S, Chikuda H, Ohya J, et al. Risk of infectious complications associated with blood transfusion in elective spinal surgery-a propensity score matched analysis. Spine J 2016;16(01):55-60

6 Kumar N, Chen Y, Nath C, Liu EH. What is the role of autologous blood transfusion in major spine surgery? Am J Orthop 2012;41 (06):E89-E95

7 Blanchette CM, Wang PF, Joshi AV, Asmussen M, Saunders W, Kruse P. Cost and utilization of blood transfusion associated with spinal surgeries in the United States. Eur Spine J 2007;16(03):353-363

8 Cushner FD, Friedman RJ. Blood loss in total knee arthroplasty. Clin Orthop Relat Res 1991;(269):98-101

9 Sehat KR, Evans R, Newman JH. How much blood is really lost in total knee arthroplasty? Correct blood loss management should take hidden loss into account. Knee 2000;7(03):151-155

10 Engoren M, Mitchell E, Perring P, Sferra J. The effect of erythrocyte blood transfusions on survival after surgery for hip fracture.J Trauma 2008;65(06):1411-1415

11 Bierbaum BE, Callaghan JJ, Galante JO, Rubash HE, Tooms RE, Welch $\mathrm{RB}$. An analysis of blood management in patients having a total hip or knee arthroplasty. J Bone Joint Surg Am 1999;81(01):2-10

12 Noticewala MS, Nyce JD, Wang W, Geller JA, Macaulay W. Predicting need for allogeneic transfusion after total knee arthroplasty. J Arthroplasty 2012;27(06):961-967

13 Morcos MW, Jiang F, McIntosh G, et al. Predictors of blood transfusion in posterior lumbar spinal fusion: a Canadian Spine Outcome and Research Network Study. Spine 2018;43(01): E35-E39

14 Tse EY, Cheung WY, Ng KF, Luk KD. Reducing perioperative blood loss and allogeneic blood transfusion in patients undergoing major spine surgery. J Bone Joint Surg Am 2011;93(13):1268-1277

15 Emara WM, Moez KK, Elkhouly AH. Topical versus intravenous tranexamic acid as a blood conservation intervention for reduction of post-operative bleeding in hemiarthroplasty. Anesth Essays Res 2014;8(01):48-53

16 Lee C, Freeman R, Edmondson M, Rogers BA. The efficacy of tranexamic acid in hip hemiarthroplasty surgery: an observational cohort study. Injury 2015;46(10):1978-1982

17 Sabboubeh A, Banaszkiewicz PA, McLeod I, Ashcroft GP, Maffulli $\mathrm{N}$. Intramedullary nailing of multiple long-bone fractures of the 
lower extremity at the same surgery: a single-center experience. J Orthop Sci 2003;8(03):313-318

18 Giddie J, Sawalha S, Parker M. Retrograde nailing for distal femur fractures in the elderly. SICOT J 2015;1:31

19 Petäjä J, Myllynen P, Myllylä G, Vahtera E. Fibrinolysis after application of a pneumatic tourniquet. Acta Chir Scand 1987; 153(11-12):647-651

20 Melvin JS, Stryker LS, Sierra RJ. Tranexamic acid in hip and knee arthroplasty. J Am Acad Orthop Surg 2015;23(12):732-740

21 Benoni G, Fredin H. Fibrinolytic inhibition with tranexamic acid reduces blood loss and blood transfusion after knee arthroplasty: a prospective, randomised, double-blind study of 86 patients. J Bone Joint Surg Br 1996;78(03):434-440

22 Georgiadis AG, Muh SJ, Silverton CD, Weir RM, Laker MW. A prospective double-blind placebo controlled trial of topical tranexamic acid in total knee arthroplasty. J Arthroplasty 2013;28(8, Suppl):78-82

23 MacGillivray RG, Tarabichi SB, Hawari MF, Raoof NT. Tranexamic acid to reduce blood loss after bilateral total knee arthroplasty: a prospective, randomized double blind study. J Arthroplasty 2011; 26(01):24-28

24 Panteli M, Papakostidis C, Dahabreh Z, Giannoudis PV. Topical tranexamic acid in total knee replacement: a systematic review and meta-analysis. Knee 2013;20(05):300-309

25 Tanaka N, Sakahashi H, Sato E, Hirose K, Ishima T, Ishii S. Timing of the administration of tranexamic acid for maximum reduction in blood loss in arthroplasty of the knee. J Bone Joint Surg Br 2001;83 (05):702-705

26 Yang ZG, Chen WP, Wu LD. Effectiveness and safety of tranexamic acid in reducing blood loss in total knee arthroplasty: a metaanalysis. J Bone Joint Surg Am 2012;94(13):1153-1159

27 Johansson T, Pettersson LG, Lisander B. Tranexamic acid in total hip arthroplasty saves blood and money: a randomized, double-blind study in 100 patients. Acta Orthop 2005;76(03): 314-319

28 Niskanen RO, Korkala OL. Tranexamic acid reduces blood loss in cemented hip arthroplasty: a randomized, double-blind study of 39 patients with osteoarthritis. Acta Orthop 2005;76(06): 829-832

29 Yue C, Kang P, Yang P, Xie J, Pei F. Topical application of tranexamic acid in primary total hip arthroplasty: a randomized double-blind controlled trial. J Arthroplasty 2014;29(12):2452-2456

30 Zhou XD, Tao LJ, Li J, Wu LD. Do we really need tranexamic acid in total hip arthroplasty? A meta-analysis of nineteen randomized controlled trials. Arch Orthop Trauma Surg 2013;133(07):1017-1027
31 Shakur H, Roberts I, Bautista R, et al; CRASH-2 trial collaborators. Effects of tranexamic acid on death, vascular occlusive events, and blood transfusion in trauma patients with significant haemorrhage (CRASH-2): a randomised, placebo-controlled trial. Lancet 2010;376(9734):23-32

32 Roberts I, Shakur H, Afolabi A, et al; CRASH-2 collaborators. The importance of early treatment with tranexamic acid in bleeding trauma patients: an exploratory analysis of the CRASH-2 randomised controlled trial. Lancet 2011;377(9771):1096-1101, 1101. e1-1101.e2

33 Farrow LS, Smith TO, Ashcroft GP, Myint PK. A systematic review of tranexamic acid in hip fracture surgery. Br J Clin Pharmacol 2016;82(06):1458-1470

34 Sukeik M, Alshryda S, Haddad FS, Mason JM. Systematic review and meta-analysis of the use of tranexamic acid in total hip replacement. J Bone Joint Surg Br 2011;93(01):39-46

35 Abdel MP, Chalmers BP, Taunton MJ, et al. Intravenous versus topical tranexamic acid in total knee arthroplasty: both effective in a randomized clinical trial of 640 patients. J Bone Joint Surg Am 2018;100(12):1023-1029

36 Alshryda S, Sarda P, Sukeik M, et al. Tranexamic acid in total knee replacement: a systematic review and meta-analysis. J Bone Joint Surg Br 2011;93:1577-1185

37 Maniar RN, Kumar G, Singhi T, Nayak RM, Maniar PR. Most effective regimen of tranexamic acid in knee arthroplasty: a prospective randomized controlled study in 240 patients. Clin Orthop Relat Res 2012;470(09):2605-2612

38 Lin PC, Hsu CH, Huang CC, Chen WS, Wang JW. The blood-saving effect of tranexamic acid in minimally invasive total knee replacement: is an additional pre-operative injection effective? J Bone Joint Surg Br 2012;94(07):932-936

39 Ker K, Edwards P, Perel P, Shakur H, Roberts I. Effect of tranexamic acid on surgical bleeding: systematic review and cumulative meta-analysis. BMJ 2012;344:e3054

40 Madsen RV, Nielsen CS, Kallemose T, Husted H, Troelsen A. Low risk of thromboembolic events after routine administration of tranexamic acid in hip and knee arthroplasty. J Arthroplasty 2017;32(04):1298-1303

41 Gandhi R, Evans HM, Mahomed SR, Mahomed NN. Tranexamic acid and the reduction of blood loss in total knee and hip arthroplasty: a meta-analysis. BMC Res Notes 2013;6:184

42 Poeran J, Rasul R, Suzuki S, et al. Tranexamic acid use and postoperative outcomes in patients undergoing total hip or knee arthroplasty in the United States: retrospective analysis of effectiveness and safety. BMJ 2014;349:g4829 